\title{
Eine Analyse der Psychoanalyse
}

\section{Paul Hoff}

Chefarzt, stv. Klinikdirektor Psychiatrische Universitätsklinik Zürich, Klinik für Psychiatrie, Psychotherapie und Psychosomatik

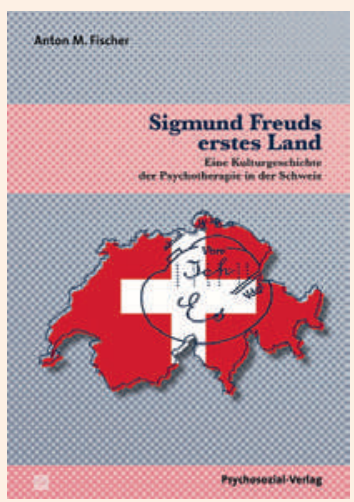

Anton M. Fischer Sigmund Freuds erstes Land Eine Kulturgeschichte der Psychotherapie in der Schweiz Giessen: Psychosozial-Verlag; 2013.

700 Seiten. $84.90 \mathrm{CHF}$ ISBN 978-3-8379-2234-9

Korrespondenz:

Prof. Dr. med. Dr. phil. Paul Hoff Psychiatrische Universitätsklinik Zürich

Lenggstrasse 31

CH-8032 Zürich

Tel. 0443842676

paul.hoff[at]puk.zh.ch
Die Schweizer Psychiatrie- und Psychotherapielandschaft ist oft zu einem Schmelztiegel unterschiedlichster Strömungen geworden. Dies ebnete den Weg nicht nur für Amalgamierungen, sondern auch für scharfe Abgrenzungen und echte Neuentwicklungen. Etwas zürichlastig sei hinzugefügt: Es macht viel Sinn, die Historie der Psychiatriestadt Zürich kritisch aufzuarbeiten und mit der heutigen Debatte zu verknüpfen. Erfreulicherweise geschieht dies in der jüngeren Vergangenheit zunehmend und auf hohem Niveau.

Mit einem landes-, ja europaweiten Kontext beschäftigt sich das vorliegende Buch. Schon die ersten Sätze der Einleitung sind Programm: «Die moderne Psychotherapie beginnt mit Sigmund Freud. Vor ihm hat niemand entdeckt, dass man das Leiden der psychisch Kranken beeinflussen kann, indem man mit ihnen redet - oder vielmehr, ihnen zuhört.» Sachlich trifft das zwar nicht zu, und es ist ein wenig polemisch -, aber so schlimm ist das auch wieder nicht. Schliesslich will der Autor sein Thema dezidiert aus psychoanalytischer Perspektive bearbeiten. Zugleich aber - und hier liegt ein Problem - bürdet er sich enorme Lasten auf: Er durchpflügt eine mehr als 100-jährige Zeitspanne (!) und schreibt dabei über den psychotherapeutischen Makrokosmos (Beispiel: «Weltgeschichte in Zürich: Freud, Bleuler und Jung», die Überschrift von Kapitel 2), den Zürcher Mikrokosmos (Beispiel: «Jungs Schüler trotzen dem Meister ein Institut $a b »$, die Überschrift des Unterkapitels 5.3) sowie die spezifisch schweizerische bzw. kantonalzürcherische Gesundheitspolitik (Beispiel: «Der Preis des Siegeszuges. Der Staat greift ein oder: Vom Ärztemonopol zum Psychologenmonopol», die Überschrift des Kapitels 6). So entsteht eine sehr interessante Mischung von Themen, aber ebenso ein ausserordentlich hoher Anspruch, der auf einen Streich wohl gar nicht eingelöst werden kann.

Eine prägnant Psychoanalyse-zentrierte «Vorgeschichte» (Kapitel 1) beschreibt quasi den Weg zu Freud. Kapitel 2 und 3 sind der frühen Zürcher Phase der Psychoanalyse gewidmet (Freud, Bleuler, Jung) sowie der zunehmend «farbigen Psychoszene» einschliesslich der Kontroversen zwischen Freud, Jung, Adler und anderen, der Internationalisierung der Jung'schen Schule und der beginnenden Spannungen zwischen psychoanalytischen und (existenz-) philosophisch fundierten Ansätzen (Freud, Binswanger, Boss). Kapitel 4 bearbeitet die Psychotherapie in der Zeit des Nationalsozialismus und kommt ausführlich auf die Rolle C. G. Jungs zu sprechen. Nach einer deutlichen Kritik an Jung wegen seiner
Nachkriegsäusserungen zum Faschismus («Die deutsche Katastrophe und ihr grandioser Schweizer Interpret») erläutert der Autor in Kapitel 5 die psychotherapeutischen Debatten der 50er und 60er Jahre mit dem Erstarken der Daseinsanalyse und den «goldenen Jahren des Psychoanalytischen Seminars Zürich». Das 6. Kapitel schliesslich widmet sich ganz der berufspolitischen Entwicklung bezüglich der nichtärztlichen Psychotherapie in der Schweiz und endet mit einer detaillierten Entstehungsgeschichte des auf den 1.4.2013 in Kraft getretenen Bundesgesetzes über die Psychologieberufe.

Einige dem Rezensenten aufgefallene Akzente seien näher erläutert.

\section{Eugen Bleuler}

Bleulers Position zur Psychoanalyse wird ausführlich gewürdigt. Die Nachkommen hatten dem Autor Einsicht in den Briefwechsel zwischen Eugen Bleuler und Sigmund Freud gewährt [1]. So wird eindrucksvoll die Ausnahmestellung Bleulers spürbar, war er doch der einzige europäische Psychiater in exponierter akademischer Stellung, der sich nicht nur intensiv mit der Psychoanalyse auseinandersetzte, sondern ihre Methoden auch konsequent in die klinische Praxis einer grossen psychiatrischen Institution, des Burghölzli, einbrachte.

Demgegenüber kommt Bleulers Grundverständnis des Faches Psychiatrie sowie seine Verankerung in der psychiatrischen Ideengeschichte des 19. Jahrhunderts kaum zur Sprache. Jedoch wäre eine solche Betrachtung hier durchaus von Interesse, etwa weil Bleuler dem (prä-psychoanalytischen) Begriff «Assoziation» bzw. «Assoziationsstörung» eine zentrale Position zuweist.

\section{Psychiatriekritischer Tenor}

Um keine Missverständnisse aufkommen zu lassen (da der Rezensent Psychiater ist): Psychiatriekritik war und ist nötig. Sie sollte sich aber unkritischer Verkürzungen enthalten. Diesem Anspruch wird der Text nicht immer gerecht.

Der psychiatrische Planer des Burghölzli, Wilhelm Griesinger (1817-1868) sowie der erste Direktor, Bernhard von Gudden (1824-1886), kommen besonders schlecht weg: Sie hätten sich für «die Insassen primär als Forschungsobjekte» interessiert, «insbesondere wenn sie tot waren und ihre Hirne endlich untersucht werden konnten.» Im Übrigen seien beide heute «völlig vergessen» (S. 22). Dass die hirnanatomische Forschung für diese Autoren wichtig war, ist zutreffend. Hingegen haben sie sich beide sehr wohl 
intensiv für praktisch-klinische Fragen eingesetzt, etwa - in heutiger Terminologie - für die sozialpsychiatrische Versorgung der Bevölkerung (Griesinger) sowie für die systematische Prävention von Zwangsmassnahmen (von Gudden).

Die Elektrokrampftherapie (EKT), häufiges Pièce de résistance, wenn es um Psychiatriekritik geht, wird als Ausdruck therapeutischer Verzweiflung in Anbetracht der «Ohnmacht gegenüber akuten Psychotikern» (S. 417) dargestellt. So etwa habe «der Psychoanalytiker [Max; P.H.] Müller [...] zuerst den Insulinschock und dann den Elektroschock populär» gemacht, «aber kaum einen Beitrag zur Weiterentwicklung der Psychoanalyse geleistet» (S. 421). Die heute empirisch überzeugend belegte Wirksamkeit der EKT bei bestimmten Indikationen bleibt unerwähnt.

Mit Blick auf psychiatrische Diagnosen ist pauschal von der «Beliebigkeit der Etiketten» die Rede, die den Betroffenen - im konkreten Fall dem Schriftsteller Friedrich Glauser (1896-1938) - «aufgeklebt wurden» (S. 421). Bei aller Kritik, die man durchaus auch als Psychiater an Diagnosen üben kann: Hier liegt eine arge Verkürzung vor.

\section{«Die Psychoanalyse ist in diesem Werk nicht nur Gegenstand, sondern auch Akteur der Untersuchung.»}

1 Eine sorgfältig kommentierte Edition des Briefwechsels zwischen Bleuler und Freud ist kürzlich erschienen: Schröter M (Hrsg.). Sigmund Freud - Eugen Bleuler. «Ich bin zuversichtlich, wir erobern bald die Psychiatrie.» Briefwechsel 1904-1937. Basel: Schwabe; 2012

2 Fischer AM. Martin Heidegger - Der gottlose Priester. Psychogramm eines Denkers. Zürich: rüffer \& rub; 2008.
Dies gilt auch für das sehr aktuelle Thema Facharztweiterbildung: «Wie bereits der FMH-Titel Spezialarzt für Psychiatrie und Psychotherapie> verrät, ist die Psychotherapie im Gesamtpaket sozusagen als Bonus inbegriffen.» (S. 555) Dabei fällt der anspruchsvolle Gegenstandskatalog der ärztlichen psychotherapeutischen Weiterbildung in der Schweiz unter den Tisch. Wie eine Ironie des Schicksals mutet es an, dass genau diese umfassende und sehr teure psychotherapeutische Weiterbildung manch interessierte(n) Kollegen/-in davon abhält, sich für das Fach Psychiatrie und Psychotherapie zu entscheiden.

\section{Nachkriegsentwicklung bis heute}

Die Entwicklung psychotherapeutischer Schulen spiegelte wie jeder gesellschaftliche Bereich die extreme Zäsur durch den Nationalsozialismus und den Zweiten Weltkrieg wider. Die nach dem Krieg erstarkte «anthropologische Psychiatrie» betrachtete psychische Erkrankungen nicht «nur» als biologische oder individuell-psychologische Phänomene, sondern als untrennbar mit der conditio humana verknüpfte Zustände.

Hier fokussiert der Autor stark auf den Einfluss Martin Heideggers, dessen Seinsphilosophie durch die Arbeiten Medard Boss' mit Fragen der praktischen Psychotherapie in Berührung kam. Doch riskiert er mit seiner berechtigten HeideggerKritik, das Kind mit dem Bade auszuschütten und den Eindruck entstehen zu lassen, (existenz-)philosophische Positionen seien hier grundsätzlich fehl am Platze. Dass dem nicht so ist, zeigen so unterschiedliche Ansätze wie die Logotherapie Viktor E. Frankls, die Gesprächspsychotherapie Carl Rogers oder neuere achtsamkeitsbasierte Psychotherapieverfahren.

\section{Stil}

Passagen mit detaillierten Beschreibungen kritischer Episoden der Psychotherapiegeschichte, die eher trocken-wissenschaftlich wirken, wechseln ab mit flüssig-erzählerischen Abschnitten, angereichert mit einer ironisch-distanzierten Note. Auch schreckt der Autor nicht vor flapsig-spöttischen, ja polemischen Tönen zurück.

Die Psychoanalyse ist in diesem Werk nicht nur Gegenstand, sondern auch Akteur der Untersuchung: Der Autor widmet spezielle Abschnitte («Streiflichter») der psychoanalytischen Interpretation zentraler Episoden der Schweizer Psychiatrieund Psychotherapiehistorie. Eine solche Verschränkung von Inhalt und Methode ist freilich nicht ohne Tücken, denn mitunter entsteht so ein recht eigenweltlicher Duktus in der Argumentation - ein Phänomen übrigens, auf das der Autor selbst an anderer Stelle kritisch hinweist, etwa mit Blick auf Kontroversen zwischen Freud und Jung.

\section{Synopsis}

Der Autor des Buches, Psychologe und - heute in eigener Praxis tätiger - Psychoanalytiker, beschäftigt sich seit langem mit ideengeschichtlichen Aspekten im facettenreichen Grenzgebiet von Psychiatrie, Psychologie, Philosophie und Gesellschaftswissenschaften. 2008 legte er eine umfassende Studie über Martin Heidegger vor [2].

Sein neues Werk ist ein sehr lesenswertes, informatives und engagiertes Buch, das inhaltlich wie methodisch eine dezidiert psychoanalytische Perspektive vorgibt. Es leuchtet manch verschlungenen Pfad der Psychotherapiegeschichte aus, ja wird mitunter zur eigentlichen Fundgrube. Das mehrheitlich kritisch-reflektierte Psychoanalyseverständnis des Autors hält sich fern von personenbezogener Hagiographie. Hingegen fallen einige Kommentare zu psychiatrischen Sach- und Personalfragen unnötig tendenziös aus. Stilistisch und inhaltlich ist der Text breit orchestriert: Er beschreibt, deutet, fragt, polarisiert; hin und wieder polemisiert er auch. Genau deswegen verdient das Buch viele neugierig-kritische Leserinnen und Leser. Sie werden es wohl nicht ohne Widerspruch, aber sicher mit Gewinn lesen. 